Title: Domain Knowledge Integration in Data Mining using Decision Tables: Case

\title{
Studies in Churn Prediction
}

\section{Elen Lima (corresponding author)}

School of Management

University of Southampton

Southampton

SO17 1BJ

United Kingdom

Tel.: +44 (0)23 80597995

Fax: +44 (0)23 80593844

E-mail: elenlima@soton.ac.uk

\section{Christophe Mues}

School of Management

University of Southampton

Southampton

SO17 1BJ

United Kingdom

Tel.: +44 (0)23 80592561

Fax: +44 (0)23 80593844

E-mail: C.Mues@soton.ac.uk

\section{Bart Baesens}

School of Management

University of Southampton

Southampton

SO17 1BJ

United Kingdom

Tel.: +44 (0)23 80598712

Fax: +44 (0)23 80593844

E-mail: Bart@soton.ac.uk 
Abstract: Companies' interest in customer relationship modelling and key issues such as customer lifetime value and churn has substantially increased over the years. However, the complexity of building, interpreting and applying these models creates obstacles for their implementation. The main contribution of this paper is to show how domain knowledge can be incorporated in the data mining process for churn prediction, viz. through the evaluation of coefficient signs in a logistic regression model, and secondly, by analysing a decision table (DT) extracted from a decision tree or rule-based classifier. An algorithm to check DTs for violations of monotonicity constraints is presented, which involves the repeated application of condition reordering and table contraction to detect counter-intuitive patterns. Both approaches are applied to two telecom data sets to empirically demonstrate how domain knowledge can be used to ensure the interpretability of the resulting models.

Keywords: domain knowledge, data mining, churn, decision tables.

\section{Introduction}

When evaluating the relationship a customer has with a company, two key concepts may be used as part of an analytical approach, customer lifetime value and churn. Customer lifetime value (CLV) (Jain and Singh, 2002; Fader et al, 2005; Malthouse and Blattberg, 2005) represents the present value of the expected benefits minus the costs of initialising, maintaining and developing the customer relationship. It involves elements such as costs, revenue, a discount rate and a time horizon. Another important aspect is retention rate or, conversely, probability of churn. As emphasised by Kim et al (2006), it is necessary to consider the churn probability in the lifetime evaluation of a customer, instead of only looking at past profit history.

Hence, the focus of this paper is to investigate churn prediction models. However, rather than simply applying data mining techniques and choosing the best predictive model, the approach we propose is to use domain knowledge (e.g. Anand et al, 1995; Alonso et al, 2002) 
integrated with data mining analysis, in order to better understand and select the best predictors that could influence the probability of a customer churning or not churning.

To be able to achieve this, we will start by examining the concept of churn and how it has been investigated in previous research. After briefly explaining some data mining techniques for churn prediction, we will elaborate on the concept of decision tables, and how they can be used to simplify and support the interpretation of rules generated by the decision tree model. Subsequently, it will be shown how domain knowledge can be integrated in the data mining analysis, i.e. through the evaluation of signs in the logistic regression and analyses of rules in a decision table. The suggested approach will then be empirically validated on two real-life data sets.

\section{The Concept of Churn}

Kim et al (2006, p. 104) describe churn as "the number or percentage of regular customers who abandon a relationship with a service provider." Some of the key studies published in the area of churn are displayed in Table 1.

All these studies use data mining or statistical methods, in order to predict the probabilities of churn. For example, Kim et al (2006) use neural networks, decision tree and logistic regression to predict churn, using the result from the best performing model in the calculation of CLV.

Neslin et al (2006) analyse the results from different data mining techniques used to calculate churn and conclude that logistic regression and decision trees performed well and are good techniques to be used when analysing churn. Larivière and Van den Poel (2004) use survival analysis to define the timing of churn; more specifically, they utilise a proportional hazard model to test the impact of cross-selling at the moment of churn likelihood so as to evaluate if this could prevent churn. 
In order to investigate how effectively support vector machines would detect churn, Kim et al (2005) compare support vector machines and back-propagation neural networks for predicting churn on a data set from a credit card company. Zao et al (2005) perform a similar analysis, comparing one-class support vector machines, neural networks, decision trees and naïve bayes, also with the intention of empirically evaluating the predictive accuracy of support vector machines. In both studies, support vector machines perform better.

However, in practice, predictive power is often not the sole criterion for model selection; ideally models should not only be accurate but also intuitive. Masand et al (1999) identify that churn models should be complemented with intuitive explanations, such that they can be successfully used by marketing analysts and decision makers. In this paper, we will address this issue by showing how to effectively incorporate domain knowledge into the churn modelling process.

\section{Data Mining Techniques for Churn Prediction}

For this research, the data mining techniques chosen were logistic regression and decision trees. The motivation is that both techniques yield simple, easy to understand models and furthermore have been shown to perform well in various problem settings, e.g. for credit scoring (Baesens et al, 2003 ${ }^{\mathrm{b}}$ ).

Logistic regression (see e.g. Allison, 2001) is a predictive modelling technique, used when the dependent variable is discrete or categorical, e.g. churn (1) or not churn (0). It uses a sigmoid transformation to limit the outcomes of the linear combination of the characteristics between 0 and 1. As such, logistic regression models the relationship between the probability of the target event occurring (e.g., churn $=1$ ) and the predictive variable (s) for the model.

Decision trees are representations which are evaluated in a top-down manner. Starting at the root node, one navigates down by evaluating the various conditions specified, until a leaf node is reached where a classification decision is made (see e.g. Figure 6) (Quinlan, 1993; 
Witten and Frank, 2005). Decision trees are built using recursive partitioning combined with information theory to measure the impurity/entropy in each node.

\section{Visualisation of Rules using Decision Tables}

Decision tables (DTs) provide an alternative way of representing rule-based classification models in a user-friendly way (Wets et al, 1997; Baesens et al, 2003 ${ }^{\mathrm{a}}$ ). They are a tabular representation used to describe and analyse decision situations, where the state of a number of conditions jointly determines the execution of a set of actions or outcomes (Vanthienen and Wets, 1994), e.g. in our context, churn or not churn. A DT consists of four quadrants, separated by thicker lines, both horizontally and vertically (Figure 1). The horizontal line divides the table into a condition part (above) and an action part (below). The vertical line separates subjects (left) from entries (right).

The condition subjects are the criteria that are relevant to the decision making process. They represent the attributes of the rule antecedents about which information is needed to classify a given customer as churner or not churner. Each condition entry describes a relevant subset of values (called a state) for a given condition subject (attribute), or contains a dash symbol ('-') if its value is irrelevant within the context of that column. Subsequently, the action entries hold the values assigned to the corresponding action subject (class), with an ' $\mathrm{x}$ ' entry indicating which value applies to a particular combination of conditions. Every column in the entry part of the DT thus comprises a classification rule. If each column only contains simple states (no contracted or irrelevant entries), the table is called an expanded DT, whereas otherwise the table is called a contracted DT. As illustrated in Figure 2, the number of columns in the contracted table can often be further minimised by changing the order of the conditions, which provides a more compact and comprehensible representation of the extracted knowledge. 
Several types of DTs have been proposed. We deliberately restrict ourselves to single-hit tables, wherein columns have to be mutually exclusive, because of their advantages with respect to verification and validation (Vanthienen et al, 1998). This type of DT can be easily checked for potential anomalies, such as inconsistencies (a particular case being assigned more than one class) or incompleteness (no class assigned).

For ease of legibility, the columns are arranged in lexicographical order, in which entries at lower condition rows alternate first. As a result, a tree structure emerges in the condition entry part of the DT, which lends itself very well to a top-down evaluation procedure. This condition-oriented inspection approach often proves more intuitive, faster, and less prone to human error, than evaluating a set of rules one by one. As a result, the DT formalism thus allows for easy validation by a practitioner or domain expert of the knowledge extracted by a rule- or tree-based classification technique (such as C4.5, CART, CHAID) against his/her own domain knowledge and prior expectations. Finally, once the DT has been approved by the expert, it can be incorporated into a deployable expert system (Vanthienen and Wets, 1994).

We will use the Prologa software to construct the DTs for the rules extracted in the empirical analyses (Vanthienen and Dries, 1994). More information about this software can be found at its website (http://www.econ.kuleuven.ac.be/Prologa/ accessed 3 September 2007).

\section{Domain Knowledge}

Domain knowledge relates to information about a specific domain or data that is collected from previous systems or documentation, or elicited from a domain expert (Anand et al, 1995). Domain knowledge can be used for discovery of meaningful information, which can then be used as a guide in the discovery process.

The approach by Anand et al (1995) combines the use of knowledge by the business expert with a discovery process that can help uncover patterns in the data that are useful for the 
analysis. They apply domain knowledge to reduce the search space before the data mining analysis, hereby making patterns more intuitive. Alonso et al (2002) use expert knowledge entered into a system not only in the model building phase, but also to clean and pre-process the data for the data mining analysis. Kopanas et al (2002) seek to identify how domain knowledge is used during different phases of the data analysis process, e.g. for data preprocessing and problem definition, where the role of the domain expert is essential.

One of the main aspects related to domain knowledge integration that is not explicitly incorporated by the three previous studies, is the monotonicity constraint (Ben-David, 1995; Sill, 1998; Feelders and Pardoel, 2003; Velikova and Daniels, 2004; Altendorf et al, 2005; Martens et al, 2006; Van Gestel et al, 2007; Martens and Baesens, 2008). A monotonicity constraint specifies that a variable has a uniquely determined impact (either increasing or decreasing) on the target. To incorporate this monotonicity requirement, different techniques have been suggested. This is for example the case for Bayesian network learning algorithms (Altendorf et al, 2005), AntMiner+ (Martens et al, 2006) and neural networks (Velikova et al, 2006).

Ben-David (1995) and Velikova and Daniels (2004) incorporate monotonicity constraints directly into the decision tree model, manipulating the resulting tree in order to obtain monotone trees. Feelders and Pardoel (2003) also test the monotonicity directly on the leaves of the classification tree, creating a large overfitted tree and pruning it towards monotone subtrees. Furthermore, Martens et al (2006) came up with the terminology of acceptable classification models for implementation, based on the idea of providing models that are accurate, comprehensible and justifiable through the use of a knowledge fusion process that incorporates domain knowledge from the experts into the data mining analysis. They are able to incorporate domain knowledge into the AntMiner+ algorithm by changing its heuristic values. 
Martens and Baesens (2008) then go on emphasising that for a classification model to be acceptable, it is necessary to check whether it is in line with existing domain knowledge, discussing how to obtain models that are comprehensible and justifiable. For them, one of the ways to obtain comprehensibility is through the construction of rule-based models, e.g. C4.5 and AntMiner+. They then introduce a metric for justifiability, in order to make it possible to compare different classifiers and also to demand a minimum value of justifiability that would make the model acceptable for implementation.

Van Gestel et al (2007) use domain knowledge for monotonicity constraint incorporation through the evaluation of signs of coefficients in the logistic regression model. Variables that present coefficients with an opposite sign to what is expected by the financial analysts are not selected for analysis. They use this variable selection as part of their analysis, considering it a key step for successful model implementation.

Verstraeten and Van den Poel (2005) propose a model to enforce signs in the logistic regression model, FSR (Forward Selection with Sign Restriction). Their model is similar to the forward selection technique, differing in the fact that the features are only included when the signs of all parameters in the model correspond to their univariate counterparts.

In this paper, we will use similar ideas to churn evaluation, by adapting the results obtained from the logistic regression and decision tree models, based on common sense and knowledge of the domain under investigation.

\section{Methodology}

Our overall methodology in explained in Figure 3. As shown, domain knowledge is first used in the pre-processing stage for making sure that the variables are well defined and meaningful from a business perspective. After the data is prepared, the logistic regression and decision tree models are constructed. To facilitate the application of domain knowledge, the signs of 
the logistic regression coefficients are evaluated and the decision tree rules are converted into a DT, in order to facilitate the detection of any counter-intuitive rules.

With regard to the logistic regression model, domain knowledge is expressed in terms of the expected sign of the variable coefficients. Next, the analysis of coefficient signs is undertaken following the method described in Figure 4. Using this procedure, inputs are added to the model based on their significance, and only inputs with the correct expected sign are kept.

For the decision tree model, DTs are used to facilitate the evaluation. They are used to check for expected effects of variables one-by-one, evaluating their monotonicity by moving them down in the condition order. This is done based on the algorithm provided in Figure 5. This algorithm allows verifying if the rules extracted from the DT are in agreement with the domain knowledge applied. After identifying the counter-intuitive rules, instead of simply changing the action entries in the decision table, the condition term would be removed altogether, and the table would be contracted accordingly. This can be motivated as follows. Suppose we have a rule 'if service calls $<3$ then churn', which is not intuitive, then there is no motivation why this cut-off would remain when changing the inequality of signs. So, instead of having two condition entries $<3$ and $\geq 3$, we would have a contracted entry '-', and the predicted class would be taken as the majority class for that subsample.

It is necessary to emphasise that checking expected signs is not a new approach, although it is new and very relevant for churn evaluation. Also, other approaches exist for including monotonicity constraints in rule-based classification models, e.g., by enforcing the constraints during algorithm learning, as in the referred AntMiner+ technique.

\section{Empirical Results}

To validate the framework presented in Figure 3, two data sets are analysed for churn prediction. The characteristics of both data sets are presented in Table 2 .

\section{Data Description}


Telecom1 is a publicly available churn data set obtained from the KDD library (http://www.datalab.uci.edu/data/mldb-sgi/data/ accessed 03 July 2006). For a full description of the data set, please refer to Larose (2005). Telecom2 is a telecom data set used in the Churn Tournament 2003, organised by Duke University. The data set and descriptive documentation were obtained from the Center for Customer Relationship Management at Duke University (http://www.fuqua.duke.edu/centers/ccrm/datasets/download.html accessed 10 May 2007).

As stated before, a first way to make the data mining analysis compliant with domain knowledge is to correct and properly pre-process the data, eliminating unknown or invalid values, and redefining variables where necessary. For example, in Telecom1, the variable "State (US)" contained 51 values; hence, coarse classification is applied, based on the odds of the target variable (churn). By grouping categorical variables into fewer categories, a more robust predictive model can be built since fewer parameters need to be estimated. The same procedure is performed for four categorical variables in Telecom 2 .

In order to further reduce the number of variables, a variable selection procedure is run. Any variable having too many missing or invalid values were eliminated from the analysis. Furthermore, we used Cramer's V statistic for the categorical variables, and t-tests for the continuous variables to identify the most predictive variables. In the end, 20 and 21 variables remained for analysis for Telecom1 and Telecom2, respectively.

\section{Data Analysis}

The data set Telecom 1 was split into training $(67 \%=3350$ observations $)$ and test $(33 \%=$ 1650 observations) sets, stratified with the same proportion of churners and not churners in both subsets (14.1\% of churners in each subset). For the decision tree evaluation, the training set was further divided into a training and a validation set $(50 \% / 50 \%)$, whereby the latter was used for pruning, in order to avoid overfitting of the tree and reducing the tree to a size that would represent the lowest misclassification rate. 
From the Telecom2 data set, we selected a sample of 10,000 customers with the same proportion of churners and not churners $(50 \% / 50 \%)$. The proportion of churners was oversampled in order to give the predictive model a better capability of detecting discriminating patterns. This sample was again used as training data for logistic regression and training and validation data for the decision tree model. A second 5,000 records sample with the same proportion of churners and not churners as the original data set was used as a test set, resulting roughly in a $67 \% / 33 \%$ training/test set split. The test set was not oversampled to provide a more realistic test set, according to a monthly churn rate of $1.8 \%$.

Both data sets were analysed using SAS 9.1 and Enterprise Miner. The resulting test set performance measures are shown in Table 3. We calculated classification accuracy (CA), sensitivity and specificity, using the Kolmogorov-Smirnov (KS) statistic for setting the cutoff, as it provides a good balance between sensitivity and specificity. The area under the ROC curve (AUC) is also used as performance measure, as it measures the ranking power regardless of the cut-off assumed.

The results obtained with the decision tree outperform logistic regression on Telecom1, whereas logistic regression provides the better model for Telecom2. However, the purpose of this research is not only to find a model with good predictive ability, but also making sure that the resulting variables are compliant with domain knowledge. Consequently, the models themselves will be assessed on their intuitiveness as well.

\section{Telecom1 - Domain Knowledge Evaluation}

For the logistic regression model, the maximum likelihood coefficient estimates are shown in Table 4. We then use domain knowledge to evaluate the coefficients' signs, analysing which kind of influence the variable has on churning. This domain knowledge is expressed in the "Expected" Column, showing the expected signs for each variable. For example, the variable 
"Cust_Serv_Calls" suggests that it influences churn positively: if the number of service calls increases, the chance of churn also increases.

Most of the variables' signs are according to what is expected, the exception being for the variables "Day Usage (Mins)" and "Evening Usage (Mins)": it is expected that an increase in the number of minutes usage would represent a decrease in the probability of churn rather than an increase (see Table 4). These variables are then eliminated one by one, and the stepwise logistic regression procedure is rerun.

After evaluating the signs, the logistic regression results (see Table 5) present coherent variables that are more in line with the domain knowledge analysis. The final performance measures will be shown later in the "Final Results" section.

The next step is to investigate the decision tree shown in Figure 6. To facilitate examining and validating its rules, the decision tree is first converted to a DT (see Table 6), which is then checked for violations against domain knowledge using the procedure described in Figure 4. Counter-intuitive rules are identified for the variable "Cust_Serv_Calls", as specified by the arrows in Table 7. It shows that in some parts of the table, if there is an increase in customer service calls, the prediction will change at some point from churn to not churn instead of viceversa. However, domain knowledge suggests that the probability of churning should increase rather than decrease with an increase in customer calls. To remedy this, different techniques can be applied, i.e. examining the selection of a different split, collapsing of a tree branch, and change of action entries.

The first option is to choose a different split based on the next best predictive variable or based on another split from the same variable, and to repeat the decision tree analysis. In our model, however, the conflicting rules remained.

Secondly, we also attempt collapsing different branches of the tree, and then repeating the decision tree analysis. After doing so, the rules are again converted into a DT model, and all 
the conditions are re-evaluated. However, the monotonicity analysis of the resulting table showed that the conflicting rules remained.

Finally, one can also directly change the action entries to make the DT in line with domain knowledge. In our case, the DT is used to change the action entries for the variable "Cust_Serv_Calls", providing consistent rules all through the DT. As explained in the methodology section, we now combine both condition entries $<3.5$ and $\geq 3.5$ into one (indicated by '-'), and the predicted class is taken as the majority class for that subsample. The resulting DT can be seen in Table 8. The new rules are then extracted and applied to the test set; the resulting performance measures will be discussed later, in the "Final Results" section.

\section{Telecom2 - Domain Knowledge Evaluation}

As modelled for Telecom1, after running the logistic regression, some variables' signs are not in accordance with domain knowledge. For example, for the variables "Average Usage (monthly)", "Average Usage (3 months)" and "Average Usage (6 months)", it would be expected that an increase in the number of minutes usage would represent a decrease in the probability of churn.

After evaluating the signs and eliminating insignificant variables, the final table of maximum likelihood coefficient estimates presents coherent variables that are more in accordance with what is expected (Table 9). See "Final Results" section for the performance measures.

The next step is to analyse the decision tree, by converting it into a DT, and then following the same procedure adopted for Telecom1. The DT analysis again revealed counter-intuitive rules, e.g. when investigating the rules relating to the variable "Average Usage (monthly)". Table 10 shows the conflicting rules, where, conversely to what was expected from the domain knowledge, the customer becomes more likely to churn if there is an increase in the average usage. 
To remedy these situations, the various options of collapsing branches and selecting different split are attempted, but, as with Telecom1, only the option of changing action entries proves successful in providing consistent rules for the variable "Average Usage (monthly)". Again, instead of having two condition entries $<22.425$ and $\geq 22.425$, we replace them with a single '-' entry, and the predicted class is taken as the majority class for that subsample.

\section{Final Results}

The resulting performance measures before and after domain knowledge integration are reported in Table 11.

Firstly, from these results, it can be concluded that logistic regression is a suitable choice of classifier for integrating domain knowledge into the model, as, in the analysis of both data sets, the model's performance stays relatively stable with the introduction of domain constraints when taking into consideration the AUC measure. Variations in performance values are still fairly small; for Telecom2, the differences in AUC between the original and amended models are statistically insignificant at the 95\% level according to the test proposed by DeLong et al (1988), whereas for Telecom1 the difference is significant at the $95 \%$ but not the $99 \%$ level.

The increases and decreases in performance noted in the logistic regression model are due to the way the cut-off was set, using the KS statistic, where the cut-off would vary from one model to the next, in order to have a balance between sensitivity and specificity. If the same cut-off would be chosen, e.g. 0.5 , such a big variation in performance would not happen. For example, for Telecom1, the classification accuracy would be $86.73 \%$ and $86.55 \%$ before and after domain knowledge analysis, respectively, but the imbalance between sensitivity and specificity would increase. Similar observations apply to Telecom2.

Secondly, for the decision tree models, we find that the incorporation of domain knowledge does not have an adverse effect on performance; instead, after making the necessary changes 
to satisfy monotonicity constraints, the decision tree for Telecom 1 even shows an improvement in performance. For both datasets, the AUC differences are not significant according to the DeLong et al (1988) test, hence showing that the proposed procedure can produce an intuitive model without sacrificing performance.

Some of these findings, in particular those for the decision tree analysis of Telecom2, are supported by other studies, e.g. by Altendorf et al (2005) and Martens et al (2006), both of which observe that, in terms of generalisation behaviour, integrating knowledge into the model does not tend to influence the final performance that much.

The difference in performance (regarding CA, sensitivity and specificity) between the logistic regression and the decision tree models, especially related to Telecom1, could also be justified in part by the chosen cut-off. If a cut-off of 0.5 was chosen, the values for the classification accuracy would be more similar (around $87 \%$ for the logistic regression model and around $90 \%$ for the decision tree model). The difference would still be significant, but different techniques could have variations in performance depending on the data, or just because one technique is better than the other in that context. As the decision tree algorithm does not make assumptions about the distribution and copes better with variable interactions, this could have influenced its better result regarding Telecom1. This can be supported by Zhao et al (2005), where different techniques presented significant variations in terms of accuracy rate. Regarding Telecom2, one can see that the performances are very similar for both models, especially before the domain knowledge evaluation. However, for both data sets, the AUC remains reasonably stable, as it is defined regardless of the cut-off established.

In conclusion, the shown procedures help to ensure that the variables presented in both models are showing the expected relationship with the target variable, with only limited loss of predictive power for logistic regression when considering the AUC performance or if 
assuming the same cut-off for the classification accuracy, and no significant loss for the decision tree models, independently of the cut-off chosen.

\section{Conclusions, Limitations and Further Research}

In this paper, we investigate how to make data mining models developed for churn prediction more understandable and compliant with domain knowledge. More specifically, we have shown how the analysis of coefficient signs in logistic regression and the monotonicity analysis of DTs can be used to check whether the knowledge contained in data mining models is in accordance with domain knowledge, and how to correct any discrepancies found.

The idea is not only to help companies discover which customers are more valuable or will churn, but to help them identify the main elements in their data that can contribute positively or negatively to the relationship with the customer, and through that, define strategies that would benefit both company and customer alike.

A limitation of the research may be in the datasets used for the empirical analysis. More specifically, in the first data set (Telecom1), the available variables were limited in that they only measure customer behaviour at one given time interval. Dynamic variables such as an increase or decrease in usage over a more extended period in time could e.g. reveal other interesting relationships with churn. The second data set (Telecom2) had a more elaborate set of variables but nonetheless presented a bigger challenge in developing an accurate model.

Further research would be interesting and useful in incorporating domain knowledge in other data mining techniques and in investigating the performance change within a technique, also analysing the reasons for any differences in performance changes. Further research could also be done in applying this approach to different data sets from the same industry so as to analyse if the behaviour of the variables would be similar, or to explore the potential of the proposed approach as a tool to support managerial or strategic decisions in settings other than churn prediction. 


\section{References}

Allison P D (2001). Logistic Regression Using the SAS System: Theory and Application. SAS Institute Inc: NC.

Alonso F, Caraça-Valente J P, González A L and Montes C (2002). Combining expert knowledge and data mining in a medical diagnosis domain. Expert Syst Appl 23(4): 367-375. Altendorf E, Restificar E and Dietterich T (2005). Learning from sparse data by exploiting monotonicity constraints. Proceedings 21st conf on Uncertainty in Artif Int, Edinburgh, Scotland.

Anand S S, Bell D A and Hughes J G (1995). The role of domain knowledge in data mining. Proceedings fourth inter conf Inf knowl Mngt Baltimore, pp 37 - 43.

Baesens B, Setiono R, Mues C and Vanthienen J (2003 $)$. Using Neural Network Rule Extraction and Decision Tables for Credit-Risk Evaluation. Mngt Sci 49(3): 312-329.

Baesens B, Van Gestel T, Viaene S, Stepanova M, Suykens J and Vanthienen J (2003 ${ }^{\mathrm{b}}$ ). Benchmarking state-of-the-art classification algorithms for credit scoring. J Opl Res Soc 54(6): $627-635$.

Ben-David A (1995). Monotonicity maintenance in information-theoretic machine learning algorithms. Machine Learning 19(1):29-43.

Buckinx W and Van den Poel D (2005). Customer base analysis: partial defection of behaviorally-loyal clients in a noncontractual FMCG retail setting. European J Opl Res 164(1): 252-268.

Burez J and Van den Poel D (2007). CRM at a pay-TV company: Using analytical models to reduce customer attrition by targeted marketing for subscription services. Expert Syst Appl 32(2): 277-288. 
Coussement K and Van den Poel D (2008). Churn prediction in subscription services: an application of support vector machines while comparing two parameter-selection techniques. Expert Syst Appl 34(1): 313-327.

DeLong E R, DeLong D M and Clarke-Pearson D L (1988). Comparing the areas under two or more correlated receiver operating curves: A nonparametric approach. Biometrics, 44(3): $837-845$.

Fader P S, Hardie B G S and Lee K L (2005). RFM and CLV: Using Iso-Value Curves for Customer Base Analysis. J Market Res 42(4): 415-430.

Feelders A and Pardoel M (2003). Pruning for monotone classification trees. In: Advanced in intelligent data analysis V: Springer, 2810: 1-12.

Hwang H, Jung T and Suh E (2004). An LTV model and customer segmentation based on customer value: a case study on the wireless telecommunication industry. Expert Syst Appl 26(2): 181-188.

Jain D and Singh S S (2002). Customer lifetime value research in marketing: A review and future directions. J Interact Market 16(2): 34-46.

Kim S, Shin K S and Park K (2005). An Application of Support Vector Machines for Customer Churn Analysis: Credit Card Case. ICNC 2005, Lect Notes Comput Sci 3611: 636647.

Kim S, Jung T, Suh E and Hwang H (2006). Customer segmentation and strategy development based on customer lifetime value: A case study. Expert Syst Appl 31(1): 101107.

Kopanas I, Avouris N M and Daskalaki S (2002). The Role of Domain Knowledge in a Large Scale Data Mining Project. In: Methods and Applications of Artificial Intelligence. Proceedings for the Second Hellenic Conference on AI, SETN 2002. Thessaloniki, pp 288299. 
Larivière B and Van den Poel D (2004). Investigating the role of product features in preventing customer churn, by using survival analysis and choice modeling: The case of financial services. Expert Syst Appl 27(2): 277-285.

Larose D T (2005). Discovering Knowledge in Data: An Introduction to Data Mining. Wiley: New Jersey.

Malthouse E C and Blattberg R C (2005). Can we predict customer lifetime value? J Interact Market 19(1): 2-16.

Martens D, De Backer M, Haesen R, Baesens B, Mues C and Vanthienen J (2006). Ant-Based approach to the knowledge fusion problem. In: Dorigo M et al (eds). ANTS Workshop 2006, Lect Notes Comput Sci 4150: 84-95.

Martens D and Baesens B (2008). Building Acceptable Classification Models, Ann Inf Syst, forthcoming.

Masand B, Datta P, Mani D R and Li B (1999). CHAMP: A Prototype for Automated Cellular Churn Prediction. Data Min Knowl Disc 3(2): 219-225.

Nath S V and Behara R S (2003). Customer churn analysis in the wireless industry: A data mining approach. Proceedings - Annual Meeting of the Decision Sciences Institute 2003: 505-510.

Neslin S A, Gupta S, Kamakura W, Lu J and Mason C (2006). Defection Detection: Measuring and Understanding the Predictive Accuracy of Customer Churn Models. J Market Res 43(2): 204-211.

Quinlan J R (1993). C4.5: programs for machine learning. Morgan Kaufmann: San Francisco, CA.

Sill J (1998). Monotonic networks. In: Advances in Neural Information Processing Systems, volume 10: The MIT Press. 
Van Gestel T, Martens D, Baesens B, Feremans D, Huysmans J and Vanthienen J (2007). Forecasting and analyzing insurance companies' ratings. Int J Forecasting 23(3): 513-529. Vanthienen J and Dries E (1994). Illustration of a decision table tool for specifying and implementing knowledge based systems. Int J Artif Int Tools 3(2): 267-288.

Vanthienen J and Wets G (1994). From decision tables to expert system shells. Data Knowl Eng 13(3): 265-282.

Vanthienen J, Mues C and Aerts A (1998). An illustration of verification and validation in the modelling phase of kbs development. Data Knowl Eng 27(3): 337-352.

Velikova M and Daniels H (2004). Decision trees for monotone price models. Comput Mngt Sci 1(3-4): 231-244.

Velikova M, Daniels H and Feelders A (2006). Solving partially monotone problems with neural networks. Proceedings of ASET 12: 82-87.

Wets G, Vanthienen J and Piramuthu S (1997). Extending a tabular knowledge based framework with feature selection. Expert Syst Appl 13(2): 109-119.

Witten I H and Frank E (2005). Data Mining: Practical machine learning tools and techniques. Morgan Kaufmann: San Francisco.

Zhao Y, Li B, Li X, Liu W, and Ren S (2005). Customer churn prediction using improved one-class support vector machine. ADMA 2005, Lect Notes Artif Int 3584: 300-306. 
Figures

\begin{tabular}{|l|l|}
\hline condition subjects & condition entries \\
\hline action subjects & action entries \\
\hline \multicolumn{2}{|c|}{ Figure 1 - DT quadrants }
\end{tabular}




\begin{tabular}{|l|c|c|c|c|c|c|c|c|}
\hline 1. InterCalls & \multicolumn{4}{|c|}{$\mathrm{Y}$} & \multicolumn{4}{c|}{$\mathrm{N}$} \\
\hline 2. Voicemail & \multicolumn{2}{|c|}{} & \multicolumn{2}{c|}{ N } & \multicolumn{2}{c|}{ Y } & \multicolumn{2}{c|}{$\mathrm{N}$} \\
\hline 3. VideoCall & $\mathrm{Y}$ & $\mathrm{N}$ & $\mathrm{Y}$ & $\mathrm{N}$ & $\mathrm{Y}$ & $\mathrm{N}$ & $\mathrm{Y}$ & $\mathrm{N}$ \\
\hline 1. Churn & - & $\mathrm{x}$ & $\mathrm{x}$ & $\mathrm{x}$ & - & $\mathrm{x}$ & - & $\mathrm{x}$ \\
\hline 2. Not Churn & $\mathrm{x}$ & - & - & - & $\mathrm{x}$ & - & $\mathrm{x}$ & - \\
\hline
\end{tabular}

(a) Expanded DT

\begin{tabular}{|l|c|c|c|c|c|}
\hline 1. InterCalls & \multicolumn{2}{|c|}{ Y } & \multicolumn{2}{c|}{ N } \\
\hline 2. Voicemail & \multicolumn{2}{|c|}{ Y } & N & \multicolumn{2}{c|}{} \\
\hline 3. VideoCall & Y & N & - & Y & N \\
\hline 1. Churn & - & $x$ & $x$ & - & $x$ \\
\hline 2. Not Churn & $\mathrm{x}$ & - & - & $\mathrm{x}$ & - \\
\hline
\end{tabular}

(b) Contracted DT

\begin{tabular}{|l|c|c|c|c|}
\hline 1. VideoCall & \multicolumn{2}{|c|}{$\mathrm{Y}$} & $\mathrm{N}$ \\
\hline 2. InterCalls & \multicolumn{2}{|c|}{$\mathrm{Y}$} & $\mathrm{N}$ & - \\
\hline 3. Voicemail & $\mathrm{Y}$ & $\mathrm{N}$ & - & - \\
\hline 1. Churn & - & $\mathrm{x}$ & - & $\mathrm{x}$ \\
\hline 2. Not Churn & $\mathrm{x}$ & - & $\mathrm{x}$ & - \\
\hline
\end{tabular}

(c) Minimised DT

Figure 2 - Minimising the number of columns of a DT 


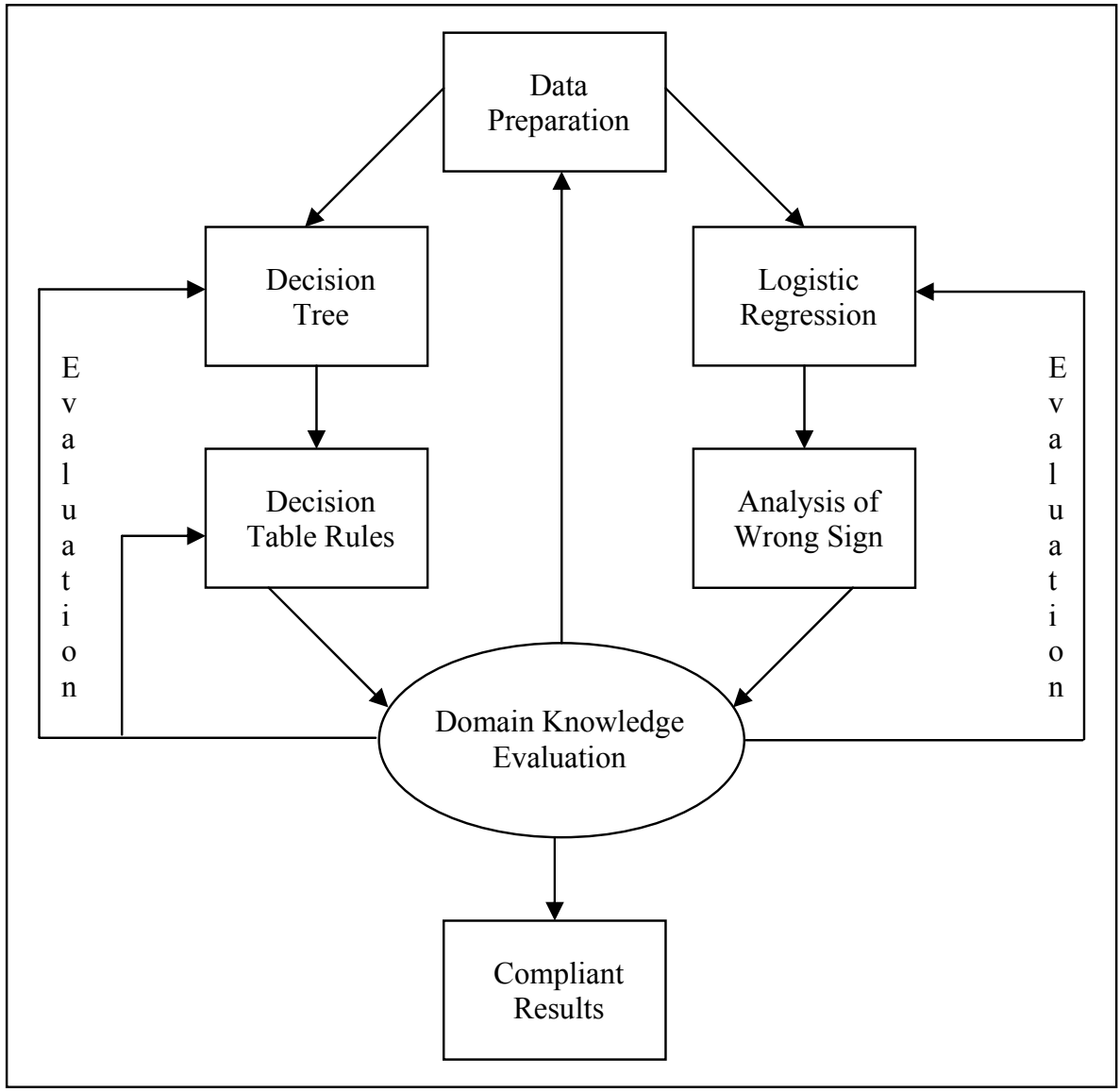

Figure 3 - Research methodology 
(1) Define domain constraint $D_{i}$ over $A_{i}$ ( $i=1, \ldots, n$, where $n$ is the number of attributes)

(2) Generate model based on stepwise regression

(3) For each attribute $A_{i}$ in the model If the coefficient sign for $A_{i}$ violates the domain constraint $D_{i}$ then Begin

Eliminate $A_{i}$ from the model: $A=A-\left\{A_{i}\right\}$

Run stepwise regression using A

Go back to (3)

End;

Figure 4 - Algorithm for selection of correct signs for logistic regression 
For each condition subject $C S_{i}(i=1, \ldots, c$, where $c$ is the number of condition subjects):

If a domain constraint $D_{i}$ has been defined over $C S_{i}$ then

Begin

Move $C S_{i}$ to last position in condition order;

Construct contracted decision table;

For each adjacent group of columns having identical condition entries for condition rows $1, \ldots, c-1$, and having condition entries for $C S_{i}$ that are different from '-', flag action value changes that violate domain constraint $D_{i}$; End;

Figure 5 - Algorithm to investigate monotonicity of a DT 


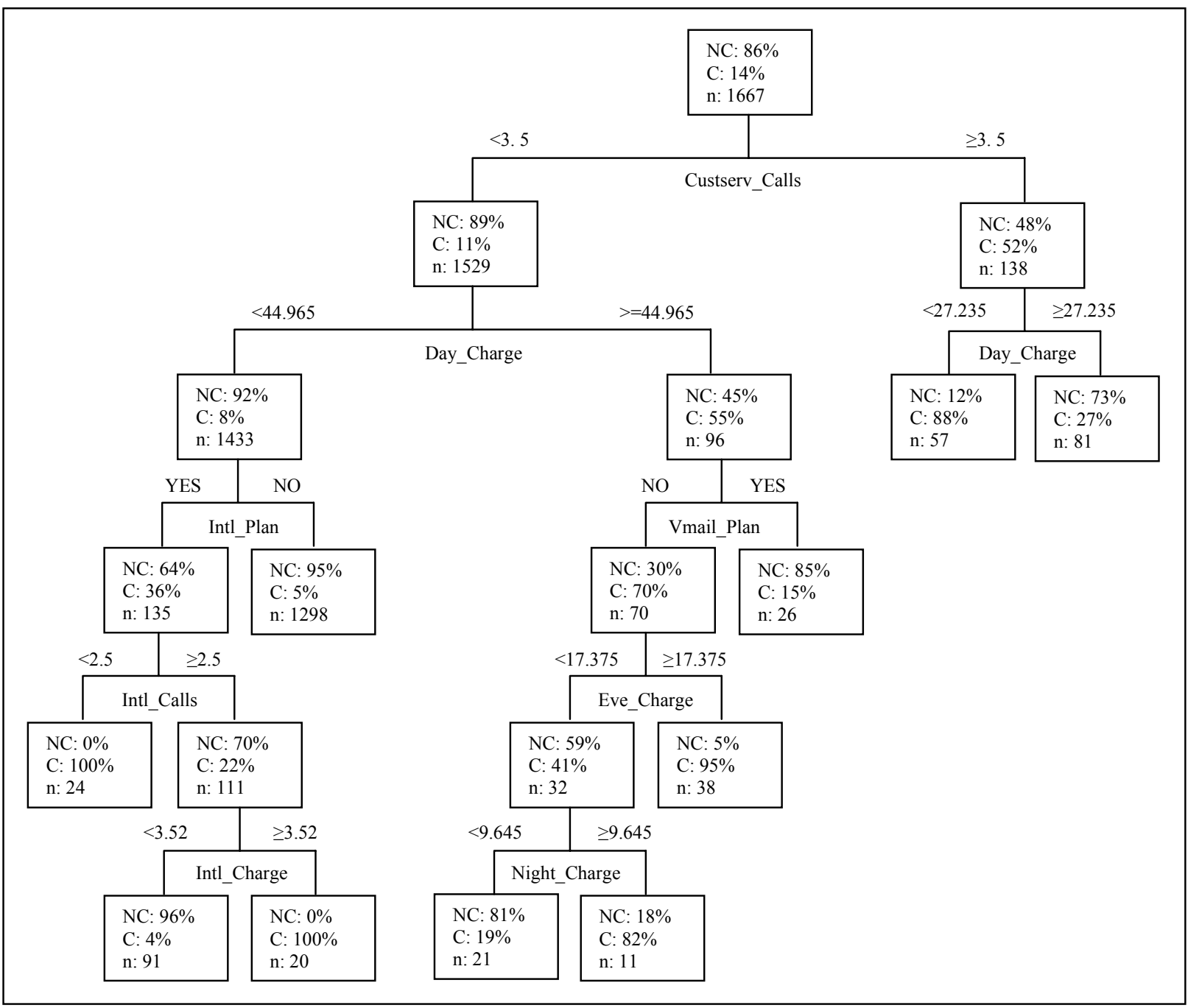

Figure 6 - Decision tree result - Telecom1 
Tables

\begin{tabular}{|l|l|}
\hline \multicolumn{1}{|c|}{ Method } & \multicolumn{1}{c|}{ Source } \\
\hline $\begin{array}{l}\text { Simple regression, nearest neighbor, decision trees and } \\
\text { neural networks }\end{array}$ & Masand et al, 1999 \\
\hline Naïve bayes and association rules & Nath and Behara, 2003 \\
\hline Neural networks, decision tree and logistic regression & Hwang et al, 2004 \\
\hline Survival analysis and proportional hazard model & $\begin{array}{l}\text { Larivière and Van den Poel, } \\
2004\end{array}$ \\
\hline SVM and back-propagation NN & Kim et al, 2005 \\
\hline $\begin{array}{l}\text { Logistic regression, ARD neural network and random } \\
\text { forests }\end{array}$ & Buckinx and Van den Poel, 2005 \\
\hline One-class SVM, NN, DT and naïve bayes & Zhao et al, 2005 \\
\hline Neural networks, decision tree and logistic regression & Kim et al, 2006 \\
\hline $\begin{array}{l}\text { Support vector machines, Logistic regression, and } \\
\text { random forests }\end{array}$ & Coussement and Van den Poel, \\
\hline $\begin{array}{l}\text { Logistic regression, decision trees, neural networks, } \\
\text { discriminant analysis, cluster analysis and bayes }\end{array}$ & Neslin et al, 2006 \\
\hline Logistic regression, markov chains and random forests & Burez and Van den Poel, 2007 \\
\hline
\end{tabular}

Table 1 - Churn literature 


\begin{tabular}{|c|c|c|c|c|c|}
\hline Data set & \# variables & \# Obs & \# Training & \# test & Churn (\%) \\
\hline Telecom1 & 20 & 5,000 & 3,350 & 1,650 & 14.1 \\
\hline Telecom2 & 21 & 15,000 & 10,000 & 5,000 & $50 / 1.8$ \\
\hline
\end{tabular}

Table 2 - Characteristics of churn data sets 


\begin{tabular}{|c|l|c|c|}
\hline Data Set & Measurement & Logistic Regression (\%) & Decision Tree (\%) \\
\hline \multirow{4}{*}{ Telecom1 } & CA & 73.65 & $\mathbf{9 0 . 6 3}$ \\
\cline { 2 - 4 } & Sensitivity & $\mathbf{8 3 . 8 3}$ & 72.34 \\
\cline { 2 - 4 } & Specificity & 71.98 & $\mathbf{9 3 . 6 4}$ \\
\cline { 2 - 4 } & AUC & 82.91 & $\mathbf{8 4 . 9 4}$ \\
\hline \multirow{5}{*}{ Telecom2 } & CA & 51.23 & $\mathbf{5 2 . 3 0}$ \\
\cline { 2 - 4 } & Sensitivity & $\mathbf{7 7 . 7 8}$ & 74.44 \\
\cline { 2 - 4 } & Specificity & 50.75 & $\mathbf{5 1 . 8 9}$ \\
\cline { 2 - 4 } & AUC & $\mathbf{6 7 . 6 4}$ & 64.25 \\
\hline
\end{tabular}

Table 3 - Original test set performance measures 


\begin{tabular}{|l|c|c|c|c|}
\hline \multicolumn{1}{|c|}{ Parameter } & Value & Expected & Estimate & P-value \\
\hline Intercept & & $+/-$ & -7.6182 & $<.0001$ \\
\hline International Plan & No & $+/-$ & -1.0588 & $<.0001$ \\
\hline Cust_Serv_Calls & & + & 0.5121 & $<.0001$ \\
\hline Day Usage (Mins) & & - & $\mathbf{0 . 0 1 2 9}$ & $<.0001$ \\
\hline Voice-mail Plan & No & $+/-$ & 0.4805 & $<.0001$ \\
\hline Evening Usage (Mins) & & - & $\mathbf{0 . 0 0 7 4 7}$ & $<.0001$ \\
\hline State (US) & Group1 & $+/-$ & -0.6261 & $<.0001$ \\
\hline International Calls & & - & -0.0982 & 0.0001 \\
\hline International Charge & & + & 0.2944 & 0.0001 \\
\hline Night Charge & & + & 0.0879 & 0.0005 \\
\hline
\end{tabular}

Table 4 - Analysis of maximum likelihood coefficient estimates - Telecom1 


\begin{tabular}{|l|l|c|c|}
\hline \multicolumn{1}{|c|}{ Parameter } & \multicolumn{1}{c|}{ Value } & Estimate & P-value \\
\hline Intercept & & -7.8545 & $<.0001$ \\
\hline International Plan & No & -1.0472 & $<.0001$ \\
\hline Cust_Serv_Calls & & 0.5152 & $<.0001$ \\
\hline Day Charge & & 0.0759 & $<.0001$ \\
\hline Voice-mail Plan & No & 0.4861 & $<.0001$ \\
\hline Evening Charge & & 0.0861 & $<.0001$ \\
\hline State (US) - G1 & No & 0.4409 & $<.0001$ \\
\hline International Charge & & 0.3122 & $<.0001$ \\
\hline International Calls & & -0.0932 & 0.0003 \\
\hline Night Charge & & 0.0880 & 0.0004 \\
\hline
\end{tabular}

Table 5 - Final analysis of maximum likelihood coefficient estimates - Telecom1 


\begin{tabular}{|c|c|c|c|c|c|c|c|c|c|c|}
\hline 1. Cust_Serv_Calls & \multicolumn{8}{|c|}{$<3.5$} & \multicolumn{2}{|c|}{$\geq 3.5$} \\
\hline 2. Day Charge & \multicolumn{4}{|c|}{$<44.965$} & \multicolumn{4}{|c|}{$\geq 44.965$} & $<27.235$ & $\geq 27.235$ \\
\hline 3. International Plan & \multicolumn{3}{|c|}{ Yes } & No & \multicolumn{4}{|c|}{-} & - & - \\
\hline 4. International Calls & $<2.5$ & \multicolumn{2}{|c|}{$\geq 2.5$} & - & \multicolumn{4}{|c|}{-} & - & - \\
\hline 5. International Charge & - & $<3.52$ & $\geq 3.52$ & - & \multicolumn{4}{|c|}{-} & - & - \\
\hline 6. Voice-mail Plan & - & - & - & - & Yes & \multicolumn{3}{|c|}{ No } & - & - \\
\hline 7. Evening Charge & - & - & - & - & - & \multicolumn{2}{|c|}{$<17.375$} & $\geq 17.375$ & - & - \\
\hline 8. Night Charge & - & - & - & - & - & $<9.645$ & $\geq 9.645$ & - & - & - \\
\hline 1. Churn & $\mathrm{x}$ & . & $\mathrm{x}$ & . & . & . & $\mathrm{x}$ & $\mathrm{x}$ & $\mathrm{x}$ & . \\
\hline 2. Not Churn & . & $\mathrm{x}$ & . & $\mathrm{x}$ & $\mathrm{x}$ & $\mathrm{x}$ & . & . & . & $\mathrm{x}$ \\
\hline
\end{tabular}

Table 6 - DT rules from the decision tree model (Figure 6) - Telecom1 


\begin{tabular}{|c|c|c|c|c|c|c|c|c|c|c|c|c|c|c|c|c|c|}
\hline 1. Day Charge & \multicolumn{6}{|c|}{$<27.235$} & \multicolumn{6}{|c|}{$(\geq 27.235)-(<44.965)$} & \multicolumn{5}{|c|}{$\geq 44.965$} \\
\hline 2. International Plan & \multicolumn{4}{|c|}{ Yes } & \multirow{2}{*}{\multicolumn{2}{|c|}{$\frac{\text { No }}{-}$}} & \multicolumn{5}{|c|}{ Yes } & No & \multirow{2}{*}{\multicolumn{5}{|c|}{-}} \\
\hline 3. International Calls & $<2.5$ & \multicolumn{3}{|c|}{$\geq 2.5$} & & & & & \multicolumn{3}{|c|}{$\geq 2.5$} & - & & & & & \\
\hline 4. International Charge & - & \multicolumn{2}{|c|}{$<3.52$} & $\geq 3.52$ & \multicolumn{2}{|c|}{-} & & & $<3.52$ & \multicolumn{2}{|c|}{$\geq 3.52$} & - & \multicolumn{5}{|c|}{-} \\
\hline 5. Voice-mail Plan & - & \multirow{2}{*}{\multicolumn{2}{|c|}{ - }} & - & \multicolumn{2}{|c|}{-} & & & - & \multicolumn{2}{|c|}{-} & - & Yes & \multicolumn{4}{|c|}{ No } \\
\hline 6. Evening Charge & - & & & - & & & & & - & & - & - & - & \multicolumn{3}{|c|}{$<17.375$} & $\geq 17.375$ \\
\hline 7. Night Charge & - & \multicolumn{2}{|c|}{-} & - & \multicolumn{2}{|c|}{-} & & & - & \multicolumn{2}{|c|}{-} & - & - & $<9.645$ & \multicolumn{2}{|c|}{$>=9.645$} & - \\
\hline 8. Cust_Serv_Calls & - & $<3.5$ & $\geq 3.5$ & - & $<3.5$ & $\geq 3.5$ & $<3.5$ & $\geq 3.5$ & - & $<3.5$ & $\geq 3.5$ & - & - & - & $<3.5$ & $\geq 3.5$ & \begin{tabular}{l|l}
$<3.5$ & $\geq 3.5$ \\
\end{tabular} \\
\hline 1. Churn & $\mathrm{x}$ & & $\mathrm{x}$ & $\mathrm{x}$ & . & $\mathrm{x}$ & $x^{4}$ & & & $\mathrm{x}$ & $\longrightarrow$ & . & . & . & $\mathrm{x}$ & $\rightarrow$ & $x \rightarrow$ \\
\hline 2. Not Churn & . & $\mathrm{x}$ & . & . & $\mathrm{x}$ & . & & $\mathrm{x}$ & $\mathrm{x}$ & & $\mathrm{x}$ & $\mathrm{x}$ & $\mathrm{x}$ & $\mathrm{x}$ & .8 & $=x$ & $4 x$ \\
\hline
\end{tabular}

Table 7 - Table 6 reordered - variable “Cust_Serv_Calls" moved to last row 


\begin{tabular}{|c|c|c|c|c|c|c|c|c|c|c|c|c|c|c|}
\hline 1. Day Charge & \multicolumn{6}{|c|}{$<27.235$} & \multicolumn{4}{|c|}{$(\geq 27.235)$ and $(<44.965)$} & \multicolumn{4}{|c|}{$\geq 44.965$} \\
\hline 2. International Plan & \multicolumn{4}{|c|}{ Yes } & \multirow{2}{*}{\multicolumn{2}{|c|}{$\frac{\text { No }}{-}$}} & \multicolumn{3}{|c|}{ Yes } & No & \multirow{2}{*}{\multicolumn{4}{|c|}{-}} \\
\hline 3. International Calls & $<2.5$ & \multicolumn{3}{|c|}{$\geq 2.5$} & & & $<2.5$ & \multicolumn{2}{|c|}{$\geq 2.5$} & - & & & & \\
\hline 4. International Charge & - & & & $\geq 3.52$ & & & - & $<3.52$ & $\geq 3.52$ & - & \multicolumn{4}{|c|}{-} \\
\hline 5. Voice-mail Plan & - & \multicolumn{2}{|c|}{-} & - & \multicolumn{2}{|c|}{-} & - & - & - & - & Yes & \multicolumn{3}{|c|}{ No } \\
\hline 6. Evening Charge & - & \multirow{2}{*}{\multicolumn{2}{|c|}{ - }} & - & \multicolumn{2}{|c|}{-} & - & - & - & - & - & \multicolumn{2}{|c|}{$<17.375$} & $\geq 17.375$ \\
\hline 7. Night Charge & - & & & - & & & - & - & - & - & - & $<9.645$ & $\geq 9.645$ & - \\
\hline 8. Cust_Serv_Calls & - & $<3.5$ & $\geq 3.5$ & - & $<3.5$ & $\geq 3.5$ & - & - & - & - & - & - & - & - \\
\hline 1. Churn & $\mathrm{x}$ & . & $\mathrm{x}$ & $\mathrm{x}$ & & $\mathrm{x}$ & $\mathbf{x}$ &. & $\mathbf{x}$ & 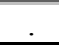 & ( & . & $\mathbf{x}$ & $\mathbf{x}$ \\
\hline 2. Not Churn & & $\mathrm{x}$ & & & $\mathrm{x}$ & & & $\mathrm{x}$ & & $\mathrm{x}$ & $\mathrm{x}$ & $\mathrm{x}$ & . & - \\
\hline
\end{tabular}




\begin{tabular}{|l|l|c|c|c|}
\hline \multicolumn{1}{|c|}{ Parameter } & Value & Expected & Estimate & P-value \\
\hline Intercept & & & -0.6106 & $<.0001$ \\
\hline Handsets (age) & & + & 0.00112 & $<.0001$ \\
\hline Mean Usage (monthly) & & - & -0.00058 & $<.0001$ \\
\hline Handset (refurb/new) & $\mathrm{N}$ & - & -0.1830 & $<.0001$ \\
\hline Handsets (price) & $\mathrm{G} 1$ & $+/-$ & -0.2176 & $<.0001$ \\
\hline Mean Revenue (monthly) & & + & 0.00355 & $<.0001$ \\
\hline N. of Handsets & & + & 0.0566 & $<.0001$ \\
\hline Credit Class & $\mathrm{G} 1$ & $+/-$ & -0.3677 & $<.0001$ \\
\hline Credit Class & $\mathrm{G} 2$ & $+/-$ & -0.2986 & 0.0003 \\
\hline Geographic_Area & $\mathrm{G} 1$ & $+/-$ & -0.1017 & $<.0001$ \\
\hline Mean Dropped Calls & & + & 0.0100 & 0.0044 \\
\hline
\end{tabular}

Table 9 - Final analysis of maximum likelihood coefficient estimates - Telecom2 


\begin{tabular}{|c|c|c|c|c|c|c|c|}
\hline 1. Handset (age) & \multicolumn{3}{|c|}{$<467.5$} & \multicolumn{4}{|c|}{$\geq 467.5$} \\
\hline 2. Handset (price) & \multicolumn{2}{|c|}{ G3 } & G2, G1 & \multicolumn{4}{|c|}{-} \\
\hline 3. Handset Web Capability & WC,NA & WCMB & - & WCMB,WC & & NA & \\
\hline 4. N. kids 3 5years & - & - & - & - & Y,UK & & \\
\hline 5. Average Usage (monthly) & - & - & - & - & - & $<22.425$ & $\geq 22.425$ \\
\hline 1. Churn & $\mathrm{x}$ & . & . & $\mathrm{x}$ & $\mathrm{x}$ & 4 & 1 \\
\hline 2. Not Churn & . & $\mathrm{x}$ & $\mathrm{x}$ & . & . & $\mathrm{x}$ & $t$ \\
\hline
\end{tabular}

Table 10 - DT rules analysis of variable “Average Usage” - Telecom2 


\begin{tabular}{|l|l|c|c|c|c|}
\hline Data Set & \multirow{2}{*}{ Measurement (\%) } & \multicolumn{2}{c|}{ Logistic Regression } & \multicolumn{2}{c|}{ Decision Tree } \\
\cline { 3 - 6 } & & Original & Final & Original & Final \\
\hline \multirow{5}{*}{ Telecom1 } & CA & 73.65 & 70.35 & 90.63 & 93.46 \\
\cline { 2 - 6 } & Sensitivity & 83.83 & 82.13 & 72.34 & 65.53 \\
\cline { 2 - 6 } & Specificity & 71.98 & 68.41 & 93.64 & 98.04 \\
\cline { 2 - 6 } & AUC & 82.91 & 80.82 & 84.94 & 86.35 \\
\hline \multirow{5}{*}{ Telecom2 } & CA & 51.23 & 64.06 & 52.30 & 52.28 \\
\cline { 2 - 6 } & Sensitivity & 77.78 & 64.44 & 74.44 & 74.44 \\
\cline { 2 - 6 } & Specificity & 50.75 & 64.06 & 51.89 & 51.87 \\
\cline { 2 - 6 } & AUC & 67.64 & 65.20 & 64.25 & 64.09 \\
\hline
\end{tabular}

Table 11 - Original and final test set performance measures (after domain knowledge analysis) 


\section{Captions for Figures and Tables}

Figure 1 - DT quadrants

Figure 2 - Minimising the number of columns of a DT

Figure 3 - Research methodology

Figure 4 - Algorithm for selection of correct signs for logistic regression

Figure 5 - Algorithm to investigate monotonicity of a DT

Figure 6 - Decision tree result - Telecom1

Table 1 - Churn literature

Table 2 - Characteristics of churn data sets

Table 3 - Original test set performance measures

Table 4 - Analysis of maximum likelihood coefficient estimates - Telecom1

Table 5 - Final analysis of maximum likelihood coefficient estimates - Telecom1

Table 6 - DT rules from the decision tree model (Figure 6) - Telecom1

Table 7 - Table 6 reordered - variable "Cust_Serv_Calls" moved to last row

Table 8 - DT rules with condition terms removed for variable "Cust_Serv_Calls" Telecom1

Table 9 - Final analysis of maximum likelihood coefficient estimates - Telecom2

Table 10 - DT rules analysis of variable "Average Usage" - Telecom2

Table 11 - Original and final test set performance measures (after domain knowledge analysis) 